\title{
Inelastic neutron scattering study and Hubbard model description of the antiferromagnetic tetrahedral molecule $\mathrm{Ni}_{4} \mathrm{Mo}_{12}$
}

\author{
J. Nehrkorn ${ }^{1}$, M. Höck ${ }^{2}$, M. Brüger ${ }^{3}$, H. Mutka ${ }^{4}$, J. Schnack ${ }^{2}$, and O. Waldmann ${ }^{1}$ \\ 1 Physikalisches Institut, Universität Freiburg, Hermann-Herder-Str. 3, D-79104 Freiburg, Germany \\ 2 Universität Bielefeld, Fakultät für Physik, Postfach 100131, D-33501 Bielefeld, Germany \\ 3 Universität Osnabrück, Fachbereich Physik, D-49069 Osnabrück, Germany \\ 4 Institut Laue-Langevin, 6 Rue Jules Horowitz, BP 156, F-38042 Grenoble Cedex 9, France
}

Received: date / Revised version: date

\begin{abstract}
The tetrameric $\mathrm{Ni}(\mathrm{II})$ spin cluster $\mathrm{Ni}_{4} \mathrm{Mo}_{12}$ has been studied by INS. The data were analyzed extensively in terms of a very general spin Hamiltonian, which includes antiferromagnetic Heisenberg interactions, biquadratic 2-spin and 3-spin interactions, a single-ion magnetic anisotropy, and DzyaloshinskyMoriya interactions. Some of the experimentally observed features in the INS spectra could be reproduced, however, one feature at $1.65 \mathrm{meV}$ resisted all efforts. This supports the conclusion that the spin Hamiltonian approach is not adequate to describe the magnetism in $\mathrm{Ni}_{4} \mathrm{Mo}_{12}$. The isotropic terms in the spin Hamiltonian can be obtained in a strong-coupling expansion of the Hubbard model at half-filling. Therefore detailed theoretical studies of the Hubbard model were undertaken, using analytical as well as numerical techniques. We carefully analyzed its abilities and restrictions in applications to molecular spin clusters. As a main result it was found that the Hubbard model is also unable to appropriately explain the magnetism in $\mathrm{Ni}_{4} \mathrm{Mo}_{12}$. Extensions of the model are also discussed.
\end{abstract}

PACS. 75.50.Xx Molecular magnets - 75.10.Dg Crystal-field theory and spin Hamiltonians - 33.15.Kr Electric and magnetic moments (and derivatives), polarizability, and magnetic susceptibility

\section{Introduction}

In the recent years molecular nanomagnets have attracted huge interest because of their sometimes spectacular magnetic properties. For instance, in molecules such as $\mathrm{Mn}_{12}$ or $\mathrm{Fe}_{8}$ slow magnetic relaxation or even quantum tunneling of the magnetization have been observed [1,2,3. A general definition which includes all relevant possibilities is difficult, but in most cases molecular nanomagnets consist of magnetic metal ions with $3 \mathrm{~d}$ shells and organic ligands. Also, in most cases the magnetism is very well described by assuming localized magnetic moments, such that metal ions with quenched orbital angular momentum are described simply by their total atomic spin $\hat{\boldsymbol{S}}_{i}(i$ numbers the metal centers in the molecule). This yields a spin Hamiltonian, which typically includes a Heisenberg exchange term describing the magnetic interactions between the metal centers in a molecule, a zero-field-splitting (ZFS) term describing the magnetic anisotropy due to the ligandfield effect, and a Zeeman term accounting for the effects of an applied magnetic field [4. However, also more complicated terms may be relevant, such as DzyaloshinskyMoriya (DM) interactions, 3-spin and 4-spin exchange interactions, or higher-order ligand-field terms.

Today, molecular nanomagnets comprise a large number of magnetic molecules, and it seems fair to say that the larger a molecule is the more interesting it tends to be. Here larger means a larger number $N$ of metal centers and/or larger spin lengths $S_{i}$, i.e., a larger dimension of the Hilbert space. A larger dimension typically gives rise to a richer structure of the energy spectra and wave functions, and wherewith to more interesting magnetic phenomena. Also, technical aspects such as the calculation of magnetic observables from the spin Hamiltonian become quickly difficult, which establishes an interesting challenge by itself.

In this context, i.e., understanding the magnetic excitations in large spin clusters, interesting molecules are for instance the antiferromagnetic (AFM) molecular wheels, in which 6 to 18 exchange-coupled metal ions form rings [5, 6, 7, 8, 9, 10, 11, 12, 13, 14, 15, 16, 17, 18. These wheels enabled the observation of phenomena such as the rotation of the Néel vector [19], which is related to the tower of states in antiferromagnetics 20,21, quantized AFM spin waves [19,22, or in the larger wheels quantum tunneling of the Néel vector and the associated quantum interference effects $[23,24,25,26$. Numerically, for hexanuclear wheels the magnetism is easily calculated exactly from the spin Hamiltonian [27,28, and in part also for $\mathrm{CsFe}_{8}$ with a dimension of the Hilbert space of 1.679.616 [13]. However, for the $\mathrm{Fe}_{18}$ wheel [17, with a Hilbert space as 

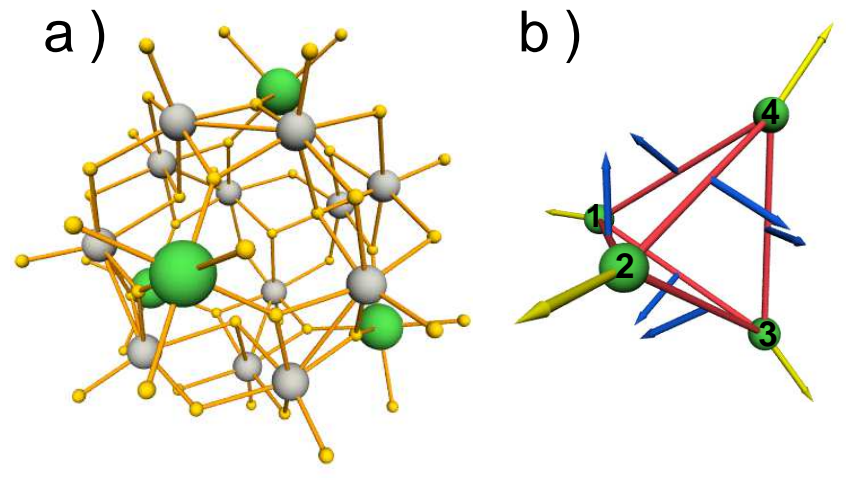

Fig. 1. (a) Ball-and-stick representation of the magnetic core of $\mathrm{Ni}_{4} \mathrm{Mo}_{12}$. Big green balls: $\mathrm{Ni}(\mathrm{II})$ ions, grey balls: Mo, small golden balls: O. $\mathrm{H}$ atoms were omitted for clarity. (b) Assumed coupling paths (red/dark sticks), orientations of the local anisotropy axes (yellow/light arrows) and DM vectors (blue/dark arrows) of $\mathrm{Ni}_{4} \mathrm{Mo}_{12}$.

large as $\approx 10^{14}$, the interpretation of the experiments requires advanced approximate techniques, which build on physical insight [26]. Another interesting molecule is the Keplerate $\mathrm{Mo}_{72} \mathrm{Fe}_{30}$ 29, in which $30 \mathrm{Fe}$ (III) ions occupy the symmetric sites of an icosidodecahedron giving rise to strong magnetic frustration effects 30 . Phenomena such as plateaus in the magnetization at $1 / 3$ magnetization due to competing spin phases 31,32 or the presence of lowlying singlets 33 were observed or predicted. The Hilbert space is huge, of dimension $\approx 10^{23}$, hence a detailed understanding of the magnetism in this cluster is obviously difficult 34,35 .

In contrast to this trend to larger molecules, sometimes even very small magnetic molecules, which at first sight would be discarded as trivial because of their small Hilbert space (which implies that "everything" can be calculated easily), may exhibit striking magnetic behavior. For instance, the $\mathrm{Fe}(\mathrm{III})$ dimer molecule $\left[\mathrm{Fe}_{2} \mathrm{~F}_{9}\left(\mathrm{Et}_{4} \mathrm{~N}\right)_{3}\right]$ exhibits unusual magnetization dynamics at low temperatures with signatures of quantum tunneling of the magnetization 36, 37, 38, the unusual magnetic behavior in the $\mathrm{Cu}(\mathrm{II})$ tetrahedron $\left[\mathrm{Cu}_{4} \mathrm{OCl}_{6}\right.$ daca $\left._{4}\right]$ was associated to phonon interactions 39 and the $\mathrm{Ni}(\mathrm{II})$ single-molecule magnet $\left[\mathrm{Ni}_{4}((\mathrm{hmp})(\mathrm{t}-\mathrm{BuEtOH}) \mathrm{Cl})_{4}\right]$ allowed one to realize quantum superpositions of high-spin states [40,41,42].

In this work we will study the tetrameric $\mathrm{Ni}(\mathrm{II})$ spin cluster $\left[\mathrm{Mo}_{12} \mathrm{O}_{30}\left(\mu_{2}-\mathrm{OH}\right)_{10} \mathrm{H}_{2}\left(\mathrm{Ni}\left(\mathrm{H}_{2} \mathrm{O}\right)_{3}\right)_{4}\right]$, or $\mathrm{Ni}_{4} \mathrm{Mo}_{12}$ in short, which is another such example [43. In $\mathrm{Ni}_{4} \mathrm{Mo}_{12}$ four $\mathrm{Ni}(\mathrm{II})$ ions occupy the vertices of an almost perfect tetrahedron and exhibit AFM nearest-neighbor Heisenberg interactions. The core of $\mathrm{Ni}_{4} \mathrm{Mo}_{12}$ is shown in Fig. 1(a). The magnetism of this "simple" cluster should be unspectacular and easy to describe. Due to the AFM Heisenberg interactions, the ground state should belong to total spin $S=0$, followed by a sequence of $S=1,2,3,4$ states with energies obeying the Landé rule $E(S)=\frac{1}{2} \Delta S(S+1)$. In an applied magnetic field, this should give rise to a series of level crossings (LCs), where the ground state changes from $S=0$ to $S=1, S=1$ to $S=2$, and so on. This sequence of LCs should in turn be detected, at low temperatures, in the magnetization curve as a sequence of steps at regular fields $B_{n}=n \Delta /\left(g \mu_{B}\right)$, with $n=1,2,3, \ldots$ [5]. For $\mathrm{Ni}_{4} \mathrm{Mo}_{12}$ magnetization steps have indeed been observed, but at fields of 4.5, 8.9, 20.1, and $32 \mathrm{~T}$, which is incompatible with the Heisenberg picture 44 . This discrepancy could not be resolved by additionally introducing ZFS and biquadratic exchange terms in the spin Hamiltonian, and a magnetic-field dependence of the exchange and ZFS parameters was hence proposed 44. Subsequently, Kostyuchenko pointed out that 3-spin interactions should not be neglected 45. Such terms can either originate from spin-phonon interactions as suggested in Ref. 44 or from electron delocalization as described by a Hubbard model 45. Indeed, the inclusion of such terms allowed Kostyuchenko to reproduce the field positions of the magnetization steps. Also, starting from a Hubbard model at half-filling, the strengths of the Heisenberg, biquadratic, and 3-spin interaction terms in the spin Hamiltonian were obtained. According to this result, the biquadratic and 3 -spin interactions should be related by a factor of 2 (in our units), which was found to be in agreement with experiment as determined from a fit of the model to the experimental field positions. This result was interpreted as to indicate the superiority of the Hubbard model for $\mathrm{Ni}_{4} \mathrm{Mo}_{12}$. More recently, Klemm and Efremov presented an extensive analysis of the magnetization in tetrameric spin clusters based on a general spin Hamiltonian [46]. However, unfortunately, the symmetry case relevant for $\mathrm{Ni}_{4} \mathrm{Mo}_{12}$ was not considered.

In order to better understand the unusual magnetism in $\mathrm{Ni}_{4} \mathrm{Mo}_{12}$, we undertook inelastic neutron scattering (INS) experiments as well as a more detailed analysis of the Hubbard model, which we will present in this work. INS is renowned for its ability to study exchange splittings in magnetic clusters directly [19,47, 48, 49,50,51,52,53. Our extensive analysis of the data in terms of a very general spin Hamiltonian will provide insight into the importance of the various interaction terms, but we could not find a parameter set which reproduces all key aspects of the data. This is certainly an unsatisfying outcome, but emphasizes the unconventionality of the magnetism in $\mathrm{Ni}_{4} \mathrm{Mo}_{12}$. It also suggests to study models going beyond the spin Hamiltonian approach, such as the Hubbard model 54, which allows for mobile electrons, i.e. itinerant magnetic moments. At half-filling the electron mobility is governed by the Hubbard- $U$ parameter, and the model of localized moments is recovered in the limit of large $U$ (strong-coupling limit). Our work on the Hubbard model is the most careful application of it to a molecular spin cluster to date. However, in contrast to Ref. 45, we find using various techniques that the Hubbard model is not adequate for describing the magnetism in $\mathrm{Ni}_{4} \mathrm{Mo}_{12}$. We will clarify this discrepancy, and as a byproduct resolve some errors in previous works.

We like to mention that a Hubbard model description of a magnetic molecule could be of interest by its own. For example, the low-energy spectrum might con- 
tain additional levels not present in a pure spin model, which may be crucial for an appropriate theoretical description of the magnetism in a molecule. As mentioned earlier, also biquadratic multiple-spin interactions such as 2-, 3-, and 4-spin terms appear in the strong-coupling limit of the Hubbard model [55]. There is thus the prospect of identifying electron delocalization as suggested by Density Functional Theory calculations 56 and modeled by the Hubbard Hamiltonian as the physical mechanism leading to such interactions and giving a better understanding of them.

The remainder of this article is organized as follows. In section 2 the experiments and a first qualitative analysis are described. Section 3 presents the phenomenological spin Hamiltonian that is used in section 4 to analyze the data with respect to various parametrizations. Section 5 discusses a possible description of $\mathrm{Ni}_{4} \mathrm{Mo}_{12}$ in terms of a Hubbard model. The article closes with conclusions.

\section{Experiments and Qualitative Analysis}

A fully-deuterated powder sample of $\mathrm{Ni}_{4} \mathrm{Mo}_{12}$ was used for the INS experiment. For details on synthesis and molecular structure see 43 . The INS data were measured on the direct time-of-flight spectrometer IN5 at the Institut Laue-Langevin (ILL, Grenoble, France). Spectra were recorded at temperatures of $2.4,9.3$, and $23 \mathrm{~K}$ for an incident neutron wavelength of $\lambda=5.0 \AA$. The energy resolution at the elastic peak was $118 \mu \mathrm{eV}$. The data were corrected for detector efficiency via a vanadium standard, and spectra were summed over all detector banks.

Fig. 2(a) presents the INS spectra. Four features can be identified on the neutron energy-loss side. At $2.4 \mathrm{~K}$ two strong peaks at about 0.4 and $0.65 \mathrm{meV}$ (peaks I and II henceforth) are clearly visible. Their intensity decreases significantly with increasing temperature. Hence, these peaks correspond to cold transitions and are magnetic in origin. At a higher energy of about $1.7 \mathrm{meV}$ (peak III) additional intensity is also visible at the lowest temperature. This broad feature is hence also assigned to a cold magnetic transition. At intermediate energies of about $1.1 \mathrm{meV}$ (peak IV) additional intensity appears at higher temperatures, which we thus assign to a hot magnetic peak. The sharp feature at $1.4 \mathrm{meV}$ marked by an asterisk in Fig. 2(a) is a spurion. The scattering intensity on the neutron energy-gain side increases with increasing temperature, in accord with general expectations and in agreement with the above assignment of the features I to IV, but is pretty featureless, which is consistent with the reduced resolution for up scattering in direct TOF instruments. We hence focus on the data on the energy-loss side in the following.

In order to be able to better analyze the features, we subtracted a background curve from the neutron energyloss data [shown as a black solid line in Fig. 2(a)], which we generated from an educated guess. Overall the true contribution from the nonmagnetic scattering should be approximated well by our curve, but it might of course
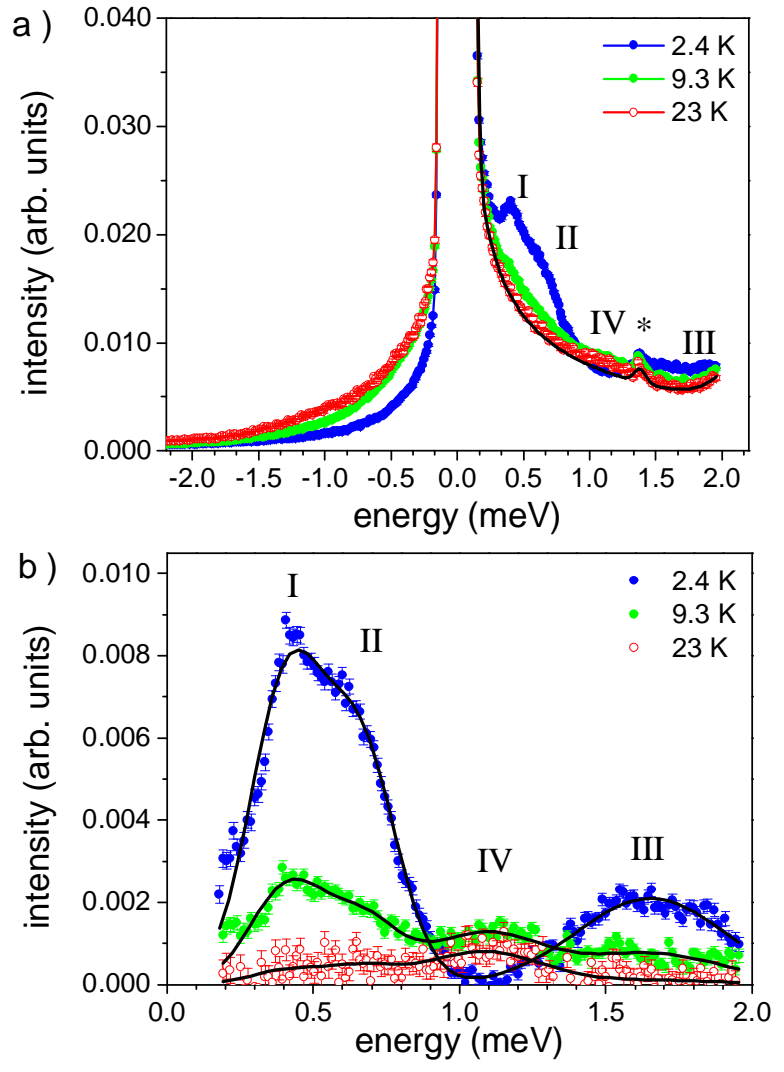

Fig. 2. (a) INS spectra of $\mathrm{Ni}_{4} \mathrm{Mo}_{12}$ at the indicated temperatures. The black line represents the background we chose (see text). (b) The INS spectra with the background subtracted. The black lines are multi-Gauss fits with parameters given in Table 1

Table 1. Results of a multi-Gauss fit analysis of the INS spectra of $\mathrm{Ni}_{4} \mathrm{Mo}_{12}$ discussed in the text. The peak positions (in $\mathrm{meV}$ ), line widths (FWHM, in meV), and peak intensities (in $10^{-3}$ arb. units) of the fitted Gauss curves are listed.

\begin{tabular}{ccccc}
\hline & I & II & III & IV \\
\hline energy & $0.408(3)$ & $0.663(4)$ & $1.65(1)$ & $1.08(5)$ \\
FWHM & 0.15 & 0.15 & $0.30(2)$ & $0.23(2)$ \\
intensity @ 2.4 K & $2.26(4)$ & $1.74(4)$ & $1.33(5)$ & 0 \\
intensity @ 9.3 K & $0.73(2)$ & $0.47(2)$ & $0.48(3)$ & $0.60(3)$ \\
intensity @ 23 K & $0.11(2)$ & $0.12(2)$ & $0.07(3)$ & $0.39(2)$ \\
\hline
\end{tabular}

be incorrect regarding finer details. The background corrected spectra are plotted in Fig. 2(b), showing the four features I to IV more clearly. Multi-Gauss fits to the peaks were done for each temperature, and the obtained peak positions, widths, and intensities are compiled in Table 1 .

The INS data indicates the energy level structure shown in Fig. 3. The cold peaks I, II, and III are assigned to transitions from the ground state to three levels at 0.4, 0.65, and $1.7 \mathrm{meV}$. The energy of peak IV matches well the gap between the two lowest excited levels and the level at $1.7 \mathrm{meV}$, which suggests to assign it accordingly. However, the temperature dependence of its intensity is maximal at around $10-15 \mathrm{~K}$, such that this peak should in fact orig- 


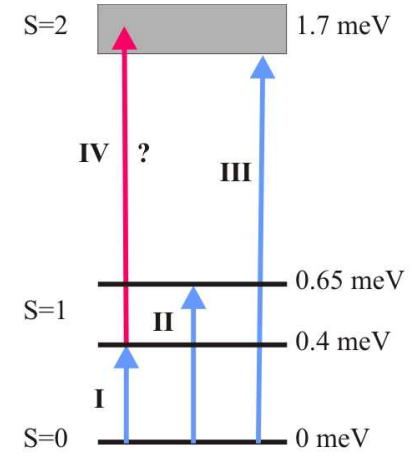

Fig. 3. Energy level structure as deduced from the INS data. The assignment of the levels to total spin quantum numbers is discussed in the text.

inate from another higher lying level. The line widths of peak I and II are somewhat larger but close to the experimental resolution, which suggests that they are made up of single transitions (broadened by e.g. $J$ strain). However, peak III is significantly broadened, which indicates that a band of energy levels exists at $1.7 \mathrm{meV}$. The width of peak IV is also larger than the experimental resolution, but in view of the weakness of the signal conclusions are not obvious.

A first attempt towards the interpretation of the INS data can be made by comparison to the magnetization data. In the magnetization curve at low temperatures four steps were observed at fields of 4.5, 8.9, 20.1, and $32 \mathrm{~T}$ [44. In a Heisenberg-exchange picture these emerge from the Zeeman splitting of the lowest multiplets for each total spin $S=0,1,2,3,4$, as described in the introduction. Since the Zeeman splitting of the involved states is known to be $-g \mu_{B} S B$, the energy of the lowest spin states in zero field can directly be determined from the magnetization steps. This yields that in zero field the lowest $S=1, \ldots, 4$ levels should be at energies of $0.6,1.7,4.4$, and $8.5 \mathrm{meV}$ (the ground state is $S=0$ ). Comparing these values to the INS findings immediately suggests to assign the peaks I and II to the $S=1$ level, with a ZFS of its $M=0$ and $M= \pm 1$ components of $0.25 \mathrm{meV}$, and peak III to the $S=2$ level, with a weaker ZFS giving rise to an enhanced line width. This assignment to spin levels is also indicated in Fig. 3 .

Energy wise, this interpretation is satisfying as it is perfectly consistent with the observed magnetization curve. However, it is inconsistent with basic rules governing INS intensity in two instances: (1) Peak III would correspond to a transition from a $S=0$ to a $S=2$ level, which is forbidden by the INS selection rule $\Delta S=0, \pm 1$. (2) The $S=1$ levels would already be thermally populated at a temperature of $2.4 \mathrm{~K}$ (we estimate a thermal population of ca. $15 \%$ ) and significant scattering intensity should be observed at this temperature, in disagreement with experiment. The above interpretation has thus to be abandoned as too simple. The behavior of peak III will in fact turn out to be a major obstacle for all the models discussed in this work.

\section{Phenomenological Spin Hamiltonian}

In view of the findings in previous works [44, 45, 57] and the above INS data, the spin Hamiltonian for $\mathrm{Ni}_{4} \mathrm{Mo}_{12}$ must include additional non-Heisenberg interactions. Here we consider the interactions

$$
\begin{aligned}
\widehat{\mathcal{H}}_{e x} & =-J \sum_{i<j}^{4} \hat{\boldsymbol{S}}_{i} \cdot \hat{\boldsymbol{S}}_{j}, \\
\widehat{\mathcal{H}}_{2} & =-J_{2} \sum_{i<j}^{4}\left(\hat{\boldsymbol{S}}_{i} \cdot \hat{\boldsymbol{S}}_{j}\right)^{2}, \\
\widehat{\mathcal{H}}_{3} & =-J_{3} \sum_{i \neq j \neq k}^{4}\left(\hat{\boldsymbol{S}}_{i} \cdot \hat{\boldsymbol{S}}_{j}\right)\left(\hat{\boldsymbol{S}}_{j} \cdot \hat{\boldsymbol{S}}_{k}\right), \\
\widehat{\mathcal{H}}_{D M} & =-\sum_{i<j}^{4} \boldsymbol{d}_{i j} \cdot\left(\hat{\boldsymbol{S}}_{i} \times \hat{\boldsymbol{S}}_{j}\right), \\
\widehat{\mathcal{H}}_{Z F S} & =\sum_{i=1}^{4} \hat{\boldsymbol{S}}_{i} \cdot \boldsymbol{D}_{i} \cdot \hat{\boldsymbol{S}}_{i},
\end{aligned}
$$

where $\widehat{\mathcal{H}}_{\text {ex }}$ describes the AFM nearest-neighbor Heisenberg interactions $(J<0), \widehat{\mathcal{H}}_{2}$ and $\widehat{\mathcal{H}}_{3}$ are the isotropic fourth-order exchange terms linking 2 and 3 spins, respectively, $\widehat{\mathcal{H}}_{D M}$ describes the antisymmetric DM interactions [58,59], and $\widehat{\mathcal{H}}_{Z F S}$ describes the ZFS due to the local onsite magnetic anisotropy. To avoid confusion later on, we repeat that $\hat{\boldsymbol{S}}_{i}$ denotes the spin operator of the $i$ th $\mathrm{Ni}(\mathrm{II})$ ion $\left(S_{i}=1\right.$ for all $\left.i\right)$.

In principle, if going to fourth order in the isotropic exchange then also a term linking 4 spins should be included. Its general structure is $\left(\hat{\boldsymbol{S}}_{i} \cdot \hat{\boldsymbol{S}}_{j}\right)\left(\hat{\boldsymbol{S}}_{k} \cdot \hat{\boldsymbol{S}}_{l}\right)$ with four different indices $i, j, k$, and $l$. According to Ref. [45, this 4-spin contribution vanishes in the case of $\mathrm{Ni}_{4} \mathrm{Mo}_{12}$; we hence disregarded it in our analysis. Our discussion in section 5 will show that there is no reason to assume that the 4-spin interactions cancel out, but we checked that neglecting them does not affect our general conclusions.

Because of the nominal high symmetry of $\mathrm{Ni}_{4} \mathrm{Mo}_{12}$ and to avoid a further increase in the number of parameters, we assumed that the isotropic coupling constants $J, J_{2}$, and $J_{3}$ are equal for each coupling path. The Hamiltonian $\widehat{\mathcal{H}}_{e x}+\widehat{\mathcal{H}}_{2}+\widehat{\mathcal{H}}_{3}$ can be solved analytically by using the vector-coupling rules of angular momenta (Kambe's method) 45, 46. However, the eigenvalues provided in Refs. [45] and [46] disagree. We hence performed systematic test calculations using exact numerical diagonalization, which confirmed the eigenvalues in Ref. [45].

According to the DM rules [59], the DM vectors $\boldsymbol{d}_{i j}$ should vanish for a perfectly tetrahedral cluster. However, a slight distortion from this high symmetry allows for nonzero DM interactions, which can in fact become significant [59, 60. Assuming a $S_{4}$ symmetry, the orientations of the $\boldsymbol{d}_{i j}$ shown in Fig. 1(b) result, with equal lengths of the DM vectors for all coupling paths. We therefore write $\boldsymbol{d}_{i j}=$ $d \boldsymbol{e}_{i j}$ with appropriate unit vectors $\boldsymbol{e}_{i j}$. 
The on-site anisotropy terms may also be effective in a perfectly tetrahedral cluster, but the local anisotropy tensors are obviously severely constrained in their orientation and magnitude by symmetry. To avoid over-parametrization, we assumed an axial local anisotropy on each Ni site, each characterized by a tensor $\boldsymbol{D}=D \operatorname{diag}\left(-\frac{1}{3},-\frac{1}{3}, \frac{2}{3}\right)$ in the local coordinate frame. The local coordinate frames are related to the cluster coordinate frame by rotation matrices $\boldsymbol{R}\left(\alpha_{i}, \vartheta_{i}, \varphi_{i}\right)$, with the Euler angles $\alpha_{i}, \vartheta_{i}$, and $\varphi_{i}$ [61. In the cluster frame the local anisotropy tensors hence become $\boldsymbol{D}_{i}=\boldsymbol{R}\left(\alpha_{i}, \vartheta_{i}, \varphi_{i}\right) \cdot \boldsymbol{D} \cdot \boldsymbol{R}^{T}\left(\alpha_{i}, \vartheta_{i}, \varphi_{i}\right)$. The local $z$ axes were chosen to point radially outwards, as shown in Fig.1(b). In principle, any configuration of the axes which is related to this one by a global rotation would also satisfy the tetrahedral symmetry. However, such a global rotation does not affect the magnetic properties of powder samples (we neglect the weak interplay of local anisotropy and DM interactions). We mention that we also considered an additional orthorhombic on-site anisotropy, i.e., a local ZFS tensor of the form $\boldsymbol{D}=\operatorname{diag}\left(-\frac{1}{3} D+E,-\frac{1}{3} D-E, \frac{2}{3} D\right)$, but this did not lead to a significant improvement or further insight. We hence disregarded this term.

In an applied magnetic field, the Zeeman term

$$
\widehat{\mathcal{H}}_{B}=\mu_{B} g \boldsymbol{B} \cdot \sum_{i=1}^{4} \hat{\boldsymbol{S}}_{i}
$$

is additionally present, where $g$ is typically on the order of 2.3 for $\mathrm{Ni}$ (II) ions. We have neglected an anisotropy of the $g$ tensor, as it is expected to be small and irrelevant.

For a given spin Hamiltonian, the magnetization curve as well as the INS spectra were calculated numerically from the eigenpairs obtained from a full exact numerical diagonalization. For the magnetization curves, the powder average was done by numerically averaging over a grid of magnetic field orientations. The powder INS spectra were calculated using the formulae given in Refs. 62,63].

\section{Analysis}

\subsection{Heisenberg exchange plus on-site magnetic anisotropy}

The qualitative interpretation of the INS spectra in terms of a dominant Heisenberg interaction has shown that peak III would violate the INS selection rule $\Delta S=0, \pm 1$. However, for $\mathrm{Ni}(\mathrm{II})$ ions it is well known that they may exhibit on-site anisotropies as large as several ten $\mathrm{K}$ 64. Hence, the possibility arises that in $\mathrm{Ni}_{4} \mathrm{Mo}_{12}$ the anisotropy constant $D$ is on the order of or even larger than the exchange coupling $J$, which would give rise to strong mixing of spin levels, such that $S$ would cease to be a good quantum number 63, 65 66. Then also $\Delta S=0, \pm 1$ would cease to be a good selection rule. In a hope that this mechanism may explain the significant INS intensity of peak III, we did extensive simulations for the Hamiltonian $\widehat{\mathcal{H}}=\widehat{\mathcal{H}}_{e x}+\widehat{\mathcal{H}}_{Z F S}$, scanning the whole parameter regime for $J$ and $D$. This model accounts for the two most significant interaction

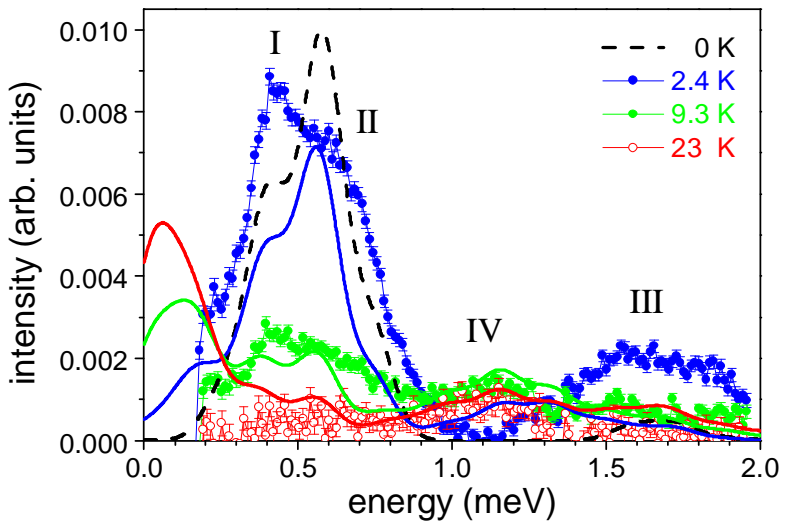

Fig. 4. Experimental (dots) and simulated (lines) INS spectra for $\widehat{\mathcal{H}}=\widehat{\mathcal{H}}_{e x}+\widehat{\mathcal{H}}_{Z F S}$ with $J=-6.5 \mathrm{~K}$ and $D=3.25 \mathrm{~K}$.

Table 2. Parameters of the three models discussed in Ref. [44].

\begin{tabular}{cccccc}
\hline model & $J^{a}$ & $J^{b}$ & $J_{2}^{a}$ & $J_{2}^{b}$ & $D$ \\
\hline $\mathrm{a}$ & $-6.4 \mathrm{~K}$ & $-6.4 \mathrm{~K}$ & $3.2 \mathrm{~K}$ & $0 \mathrm{~K}$ & $-1.0 \mathrm{~K}$ \\
$\mathrm{~b}$ & $-6.4 \mathrm{~K}$ & $-6.2 \mathrm{~K}$ & $3.0 \mathrm{~K}$ & $0 \mathrm{~K}$ & $-3.2 \mathrm{~K}$ \\
$\mathrm{c}$ & $-8.4 \mathrm{~K}$ & $-6.2 \mathrm{~K}$ & $0.32 \mathrm{~K}$ & $0.78 \mathrm{~K}$ & $-8.9 \mathrm{~K}$ \\
\hline
\end{tabular}

terms in $\mathrm{Ni}_{4} \mathrm{Mo}_{12}$. In order to be reasonably consistent with the experimental data (magnetization and INS), we observed that $D$ cannot be very large, such that the mixing effect is not very strong. Accordingly, the simulated intensity for peak III is way too weak. The best simulation of the INS spectra was obtained for $J=-6.5 \mathrm{~K}$ and $D=3.25 \mathrm{~K}$, see Fig. 4. The peak positions are well reproduced, but the discrepancies as regards the scattering intensity are obvious. Most noteworthy, the intensity of peak III is strongly underestimated and exhibits a totally wrong temperature dependence. We couldn't find any parameter set for which these discrepancies did not arise.

\subsection{The models of Schnack et al.}

Based on the magnetic susceptibility and magnetization curves, three models were suggested for $\mathrm{Ni}_{4} \mathrm{Mo}_{12}$ in Ref. [44. In these models additionally a structural distortion was accounted for via different coupling constants for the isotropic exchange, $J_{12}=J_{23}=J_{13}=J^{a}$ and $J_{14}=J_{24}=J_{34}=J^{b}$, and similarly for the 2-spin interactions, introducing parameters $J_{2}^{a}$ and $J_{2}^{b}$. The parameters of the three models are reproduced in Table2 (in our units), where the suggested magnetic field dependence of some of the parameters is irrelevant as our INS experiment was done in zero field. The simulated INS spectra are compared to the experimental data in Fig. 5 . Unfortunately, none of these models can reproduce the experimental INS spectra. The effects of DM interactions and/or tilted anisotropy tensors should be even weaker (the effect of DM interactions will be discussed in more detail in section 4.4). Hence, a deviation from the assumed tetrahedral 

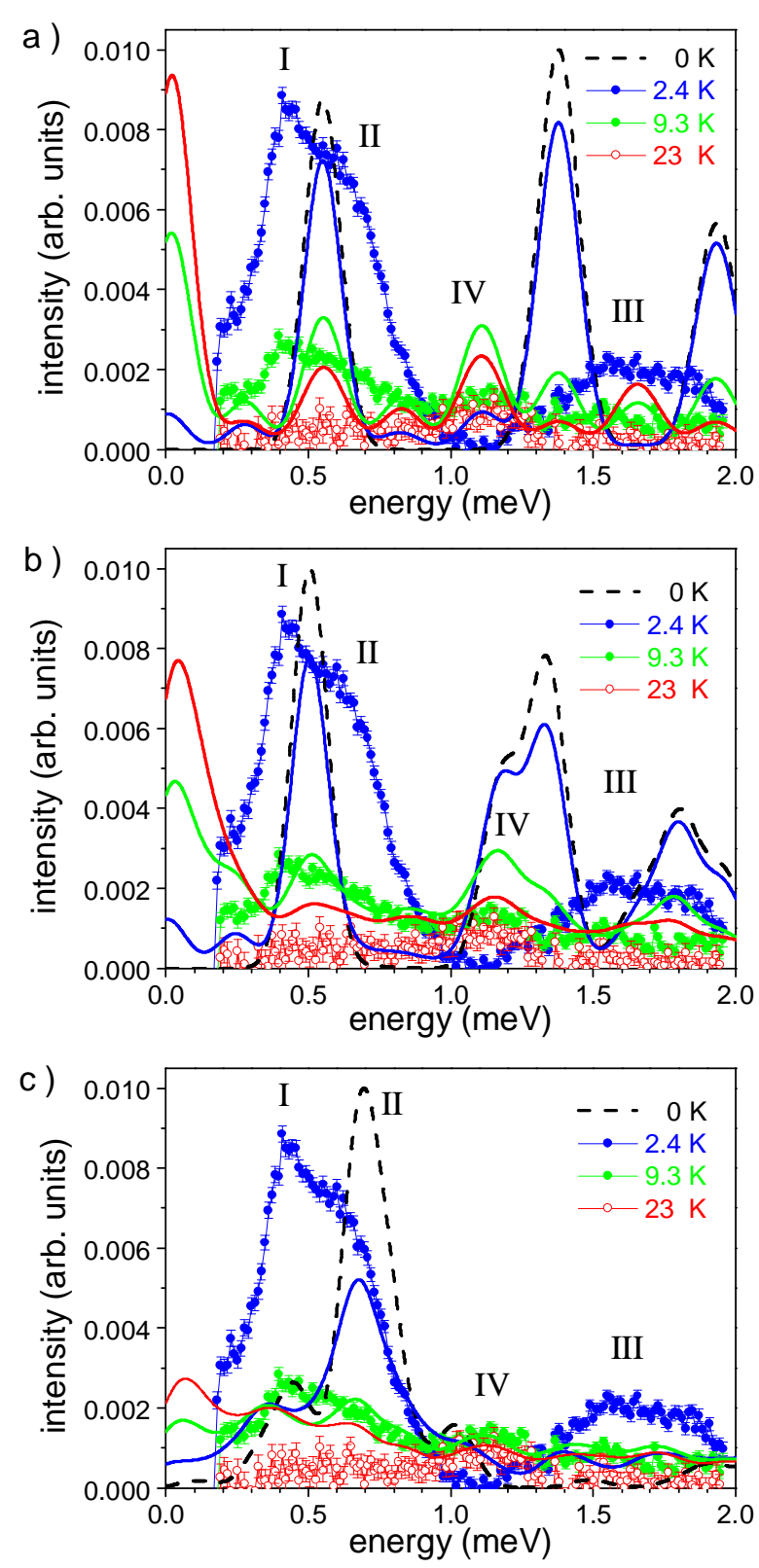

Fig. 5. Experimental (dots) and simulated (lines) INS spectra for the three models suggested in Ref. 44, see also text and Table 2

symmetry is apparently not at the heart of the unusual magnetism in $\mathrm{Ni}_{4} \mathrm{Mo}_{12}$.

\subsection{The model of Kostyuchenko}

Kostyuchenko showed recently that the positions of the steps in the magnetization curve can be reproduced very well by the model $\widehat{\mathcal{H}}=\widehat{\mathcal{H}}_{e x}+\widehat{\mathcal{H}}_{2}+\widehat{\mathcal{H}}_{3}+\widehat{\mathcal{H}}_{B}$, which in particular includes the 3 -spin interactions 45 . He also derived this spin model from the strong-coupling limit of a Hubbard model at half-filling, and found that the coupling strengths $J, J_{2}$, and $J_{3}$ are not independent. Unfortunately, as our analysis in section 5 will reveal, the

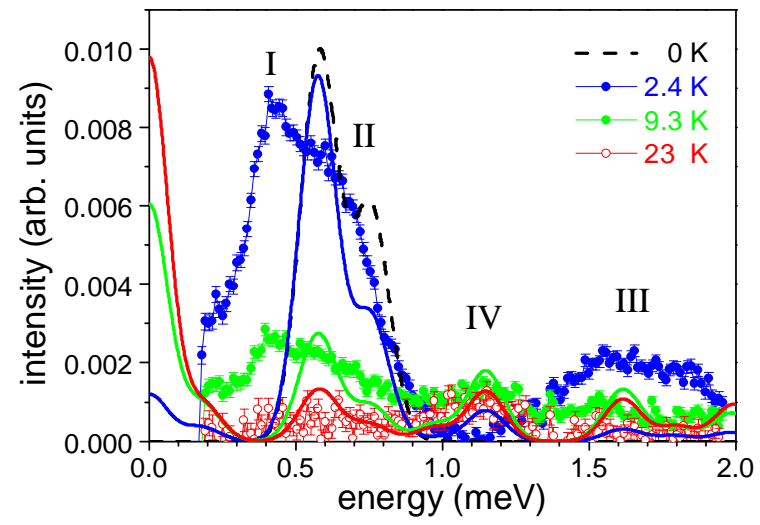

Fig. 6. Experimental (dots) and simulated (lines) INS spectra for the model suggested in Ref. 45, $\widehat{\mathcal{H}}=\widehat{\mathcal{H}}_{e x}+\widehat{\mathcal{H}}_{2}+\widehat{\mathcal{H}}_{3}$ with $J=-8.82 \mathrm{~K}, J_{2}=-1.08 \mathrm{~K}$, and $J_{3}=-0.53 \mathrm{~K}$.

obtained relations are erroneous. Nonetheless, used as a phenomenological model, $\widehat{\mathcal{H}}=\widehat{\mathcal{H}}_{e x}+\widehat{\mathcal{H}}_{2}+\widehat{\mathcal{H}}_{3}+\widehat{\mathcal{H}}_{B}$ very successfully describes the magnetization data for the parameters $J=-8.82 \mathrm{~K}, J_{2}=-1.08 \mathrm{~K}$, and $J_{3}=-0.53 \mathrm{~K}$ which were obtained from a fit to the experimentally determined crossing fields 45 . However, this model does not reproduce the experimental INS spectra well, see Fig. 6. The peak positions may be considered to be acceptable in view of the simplicity of the model, but we again observe the same discrepancy as before, i.e., the intensity of peak III is strongly underestimated and exhibits a wrong temperature dependence.

Because ZFS can be significant for $\mathrm{Ni}$ (II) ions, we extended the model to $\widehat{\mathcal{H}}=\widehat{\mathcal{H}}_{e x}+\widehat{\mathcal{H}}_{2}+\widehat{\mathcal{H}}_{3}+\widehat{\mathcal{H}}_{Z F S}+\widehat{\mathcal{H}}_{B}$ and ran a fit to the data, in which we used the magnetization and INS data simultaneously. The resulting best-fit curves are shown in Fig. 7. The magnetization is well reproduced, but the fit to the INS data shows again the "peak III discrepancy". Moreover, now also peak IV is incorrectly reproduced both as regards its intensity and temperature dependence. Apparently, also this extended model cannot satisfactorily account for peak III.

\subsection{Systematic scan of the parameter regime}

In a last effort to explain the magnetism in $\mathrm{Ni}_{4} \mathrm{Mo}_{12}$ in terms of a spin Hamiltonian we systematically considered the Hamiltonian $\widehat{\mathcal{H}}=\widehat{\mathcal{H}}_{e x}+\widehat{\mathcal{H}}_{2}+\widehat{\mathcal{H}}_{3}+\widehat{\mathcal{H}}_{Z F S}+\widehat{\mathcal{H}}_{D M}$, which includes all terms considered so far [see Eqs. (10)]. We started from simulations of the INS spectra for the Heisenberg Hamiltonian, Eq. (1a), and then extended the model by including the other terms step by step. In every step, the influence of the newly added term was analyzed. We will not discuss all details of our findings here, but just mention some key observations.

With the Heisenberg term, Eq. (1a), alone, no agreement between data and simulation could be obtained. We hence chose $J=-8.82 \mathrm{~K}$. The effects of the further terms on the INS spectra are presented in Fig. 8. Each panel 


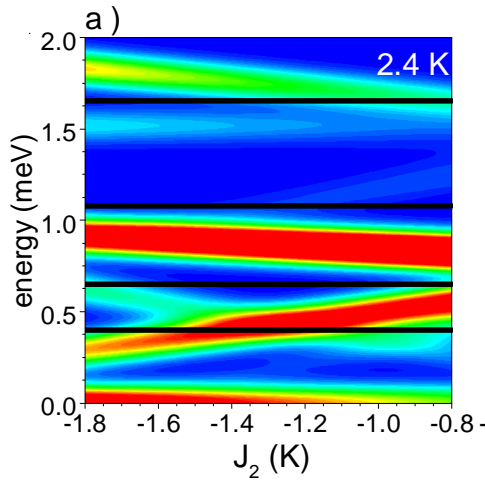

b)

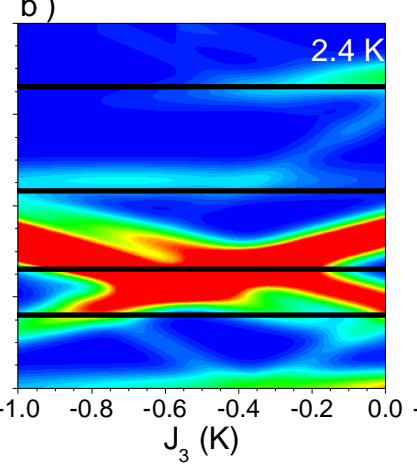

c)

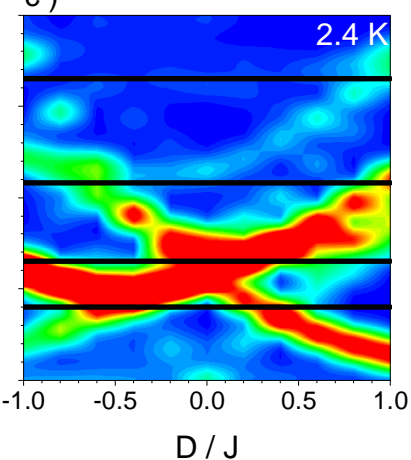

d)

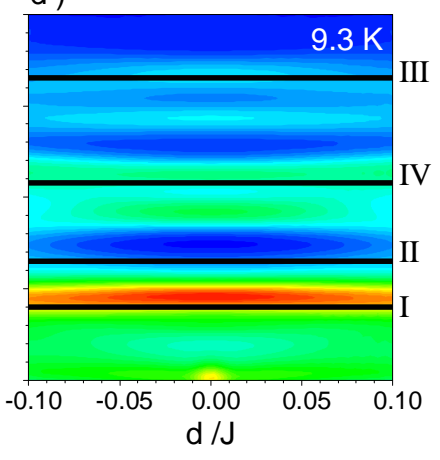

Fig. 8. Simulated INS intensity as function of energy and a selected magnetic parameter. The strength of the scattering intensity is represented by the color (red = strong, blue = weak). (a) Intensity vs. energy and $J_{2}$ at $2.4 \mathrm{~K}$. (b) Intensity vs. energy and $J_{3}$ at $2.4 \mathrm{~K}\left(J_{2}=-1.08 \mathrm{~K}\right)$. (c) Intensity vs. energy and $D$ at $2.4 \mathrm{~K}\left(J_{3}=-0.53 \mathrm{~K}\right)$. (d) Intensity vs. energy and $d$ at $9.3 \mathrm{~K}(D=3.5 \mathrm{~K})$. As a guide to the eyes, the black lines indicate the energies of the measured peaks I, II, III, and IV.
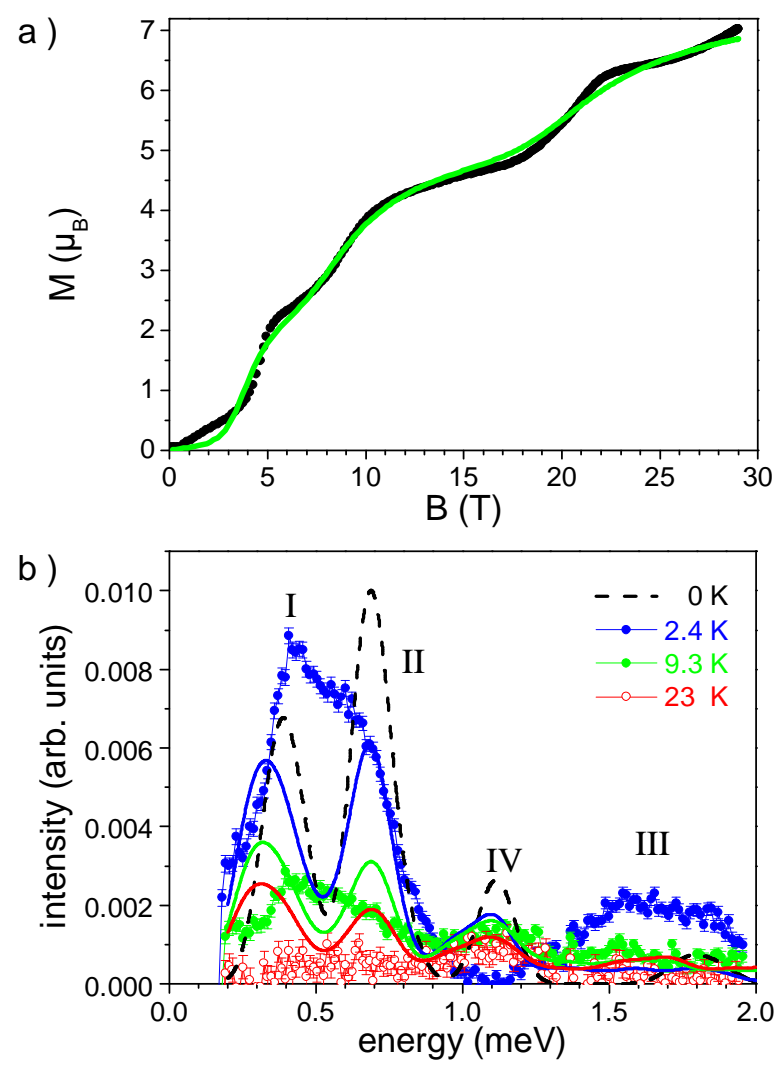

Fig. 7. (a) Experimental (black dots) and simulated (green line) magnetization curve and (b) experimental (dots) and simulated (lines) INS spectra for $\widehat{\mathcal{H}}=\widehat{\mathcal{H}}_{e x}+\widehat{\mathcal{H}}_{2}+\widehat{\mathcal{H}}_{3}+\widehat{\mathcal{H}}_{Z F S}+\widehat{\mathcal{H}}_{B}$ with $J=-9.45 \mathrm{~K}, J_{2}=-1.41 \mathrm{~K}, J_{3}=-0.66 \mathrm{~K}, D=6.26 \mathrm{~K}$, and $g=2.35$.

presents the INS intensity at one temperature, plotted as function of energy and the magnetic parameter under consideration, with the scattering intensity represented by color. The dependence of the INS intensity at $2.4 \mathrm{~K}$ on the strength of the 2-spin interaction $J_{2}$ is shown in Fig. 8(a).
Two pronounced peaks at ca. 0.5 and $0.8 \mathrm{meV}$ and a weak peak at ca. $1.8 \mathrm{meV}$ are visible. They are close to the experimental energies of features I, II, and III, but exhibit a rather weak dependence on $J_{2}$. Hence, the 2-spin interaction is important, but its exact strength is not constrained much. We chose $J_{2}=-1.08 \mathrm{~K}$. The simulations with additional 3-spin interactions are shown in Fig. 8(b). The 3 -spin interactions have a pretty strong effect on the INS spectrum. The two low-energy features, which were visible in Fig. 8(a), are now highly entangled, and the high-energy feature becomes very weak. The 3 -spin interactions were crucial for explaining the magnetization data, but as regards INS they actually have a counter productive effect. If it were not for the magnetization, one would rather abandon them in an interpretation of the INS data. We chose $J_{3}=-0.53 \mathrm{~K}$. Next the ZFS term was included; the simulations for varying $D$ values are shown in Fig. 8(c). As expected, the ZFS term has a significant effect, and for $D$ values in between $-0.5 J$ and $-0.1 J$ both peaks I and II are well reproduced. However, as discussed before, even for large $D$ values the mixing of spin levels is not strong enough to produce a significant INS intensity at the higher energies, i.e., peak III is not reproduced. Furthermore, at $2.4 \mathrm{~K}$ there is significant intensity in the energy region 0.8 to $1.2 \mathrm{meV}$, in contrast to experiment. We chose $D=-0.4 J=3.5 \mathrm{~K}$. Finally, the effect of the DM interactions on the INS spectrum at $9.3 \mathrm{~K}$ is shown in Fig. 8 (d). The DM interactions could in principle also be responsible for a mixing of spin levels and hence a violation of the INS selection rule, but apparently they have no essential effect. We therefore conclude that DM interactions are not relevant for explaining the key unexplained aspects of the magnetism in $\mathrm{Ni}_{4} \mathrm{Mo}_{12}$.

The best simulation obtained in this approach is compared to the experiment in Fig. 9. Peaks I and II are reproduced reasonably well. Also the temperature dependence of peak IV is by and large correctly obtained (increasing intensity with increasing temperature). However, as for all models discussed in this work, the intensity and temperature dependence of peak III is not reproduced. 


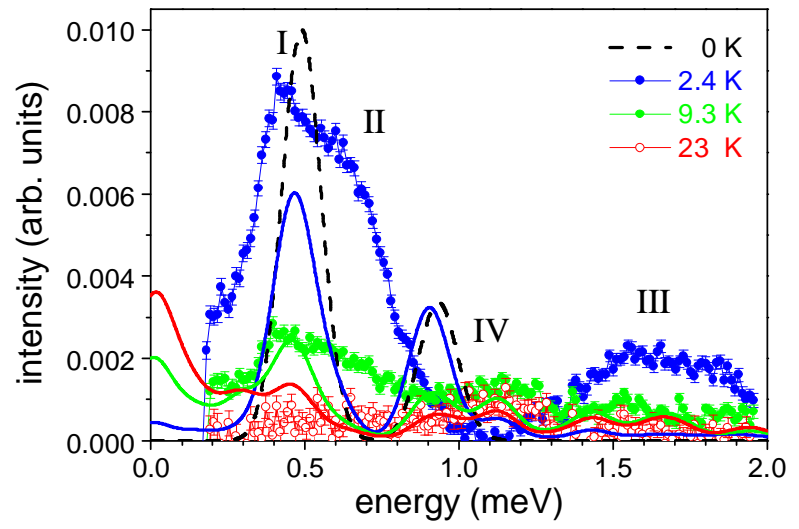

Fig. 9. Experimental (dots) and simulated (lines) INS spectra for our best-fit model, $\widehat{\mathcal{H}}=\widehat{\mathcal{H}}_{e x}+\widehat{\mathcal{H}}_{2}+\widehat{\mathcal{H}}_{3}+\widehat{\mathcal{H}}_{Z F S}$ with $J=-8.82 \mathrm{~K}, J_{2}=-1.08 \mathrm{~K}, J_{3}=-0.56 \mathrm{~K}$, and $D=3.5 \mathrm{~K}$.

To conclude this section, all our efforts to reproduce the experimental INS data with a spin Hamiltonian were unsuccessful in the sense that key aspects of the data, such as the magnitude of the intensity of peak III and its temperature dependence, could not be satisfactorily reproduced. The analysis, however, provided some insight into which terms should be most important. For example, deviations from the tetrahedral symmetry and DM interactions appear to be irrelevant. Considering the extensive yet unsuccessful spin-Hamiltonian-based efforts presented here as well as in Ref. 44, one may wonder about the apparent inability of the spin Hamiltonian approach to account for the magnetism in $\mathrm{Ni}_{4} \mathrm{Mo}_{12}$.

\section{Hubbard Model Description}

The difficulties in interpreting the experimental data by a spin Hamiltonian suggest to reanalyze its basis. A possible approach is to start with the more fundamental Hubbard model at half-filling, from which spin exchange interactions are obtained with a standard argument in a large $U$ expansion. In fact, a Hubbard model was recently proposed for $\mathrm{Ni}_{4} \mathrm{Mo}_{12}$ 45. However, as we will show in this section, this model is not adequate for describing the lowtemperature thermodynamic and spectroscopic properties of the molecule either.

As presented in Ref. [45, the motivation for applying a Hubbard model in the first place comes from the octahedral surroundings of the Ni ions which cause a splitting of their $3 d$ levels into three lower-lying $t_{2 g}$ and two higherlying $e_{g}$ orbitals. According to Hund's rules, the $t_{2 g}$ orbitals are fully occupied, whereas the $e_{g}$ orbitals are only singly occupied. The proposed Hubbard model is then formulated using a standard (one-band) Hamiltonian [54,

$$
\widehat{\mathcal{H}}_{H}=\sum_{\alpha \beta, \sigma}^{L} t_{\alpha \beta} \hat{c}_{\alpha, \sigma}^{\dagger} \hat{c}_{\beta, \sigma}+U \sum_{\alpha=1}^{L} \hat{n}_{\alpha, \uparrow} \hat{n}_{\alpha, \downarrow}+g \mu_{B} \hat{\boldsymbol{S}} \cdot \boldsymbol{B}
$$

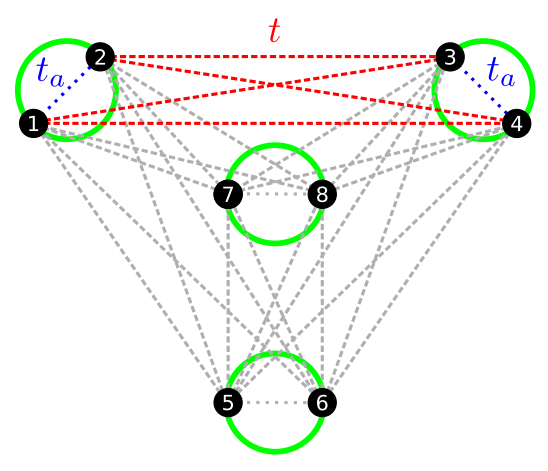

Fig. 10. Schematic representation of the lattice and the hopping paths for the Hubbard model $\widehat{\mathcal{H}}_{H}$ (cf. Fig. 2 of Ref. [4]). The small, numbered spheres correspond to Hubbard lattice sites, which are assigned to the four $\mathrm{Ni}$ ions as indicated by the large, green circles. Possible jumps of electrons are visualized by lines: inter-ion hoppings (dashed/red) occur with equal strength $t$ and intra-ion hoppings (dotted/blue) with strength $t_{a}$, but the latter are excluded and set to zero.

with parameters $N_{e}=L=8$ (half-filling), where $L$ is the number of lattice sites and $N_{e}$ the number of electrons. The $\hat{c}_{\alpha, \sigma}^{\dagger}\left(\hat{c}_{\alpha, \sigma}\right)$ are the fermionic creation (destruction) operators for an electron at lattice site $\alpha$ with spin projection $\sigma=\uparrow, \downarrow$, and $\hat{n}_{\alpha, \sigma}=\hat{c}_{\alpha, \sigma}^{\dagger} \hat{c}_{\alpha, \sigma}$. The local spin density at site $\alpha$ can be written as $\hat{\boldsymbol{s}}_{\alpha}=\sum_{\sigma \tau} \hat{c}_{\alpha, \sigma}^{\dagger} \boldsymbol{\sigma}_{\sigma \tau} \hat{c}_{\alpha, \tau}$, with the vector of Pauli matrices $\boldsymbol{\sigma}$. The total spin operator then reads $\hat{\boldsymbol{S}}=\sum_{\alpha} \hat{\boldsymbol{s}}_{\alpha}$. The possible jumps of electrons between lattice sites are characterized by the hopping parameters $t_{\alpha \beta}$, as illustrated in Fig. 10. In the considered model all hopping matrix elements corresponding to inter-ion jumps are equal to $t$, and those corresponding to intra-ion jumps are equal to $t_{a}$. The parameter $t_{a}$ is set to zero, as in Ref. 45.

In this section we will focus on the steps in the magnetization curve as predicted by the Hamiltonian $\widehat{\mathcal{H}}_{H}$. At this point we should stress that in Ref. 45 not the Hubbard model itself was analyzed, but the spin model $\widehat{\mathcal{H}}_{e x}+\widehat{\mathcal{H}}_{2}+\widehat{\mathcal{H}}_{3}+\widehat{\mathcal{H}}_{B}$ [Eqs. (1a)-(1c), and (2)], which is supposed to approximate the full Hamiltonian $\widehat{\mathcal{H}}_{H}$. In contrast, here we directly study the Hubbard model by using numerical exact diagonalization to calculate the eigenvalues of $\widehat{\mathcal{H}}_{H}$. The parameters $t$ and $U$ were then fitted to the experimentally determined crossing fields by employing a numerical minimization routine without imposing any constraints on the parameter values. Temperature was set to $T=0$, and the $g$ factor to $g=2.25$, as in Ref. 45. Despite testing a lot of initial values and initial search directions for $t$ and $U$, we only found a single, unsatisfactory fit which obeys $U>0$, namely $t=3.91 \mathrm{meV}$ and $U=64.6 \mathrm{meV}$. The predicted low-temperature magnetization curve is shown in Fig. 11. The $\chi^{2}$ value of the fit to the crossing fields is about $6.25 \mathrm{~T}^{2}$, which is approximately two orders of magnitude worse than the result obtained with the spin model $\widehat{\mathcal{H}}_{e x}+\widehat{\mathcal{H}}_{2}+\widehat{\mathcal{H}}_{3}+\widehat{\mathcal{H}}_{B}$ [45]. Considering the stability of our fit result regardless of the starting conditions, and taking into account that 


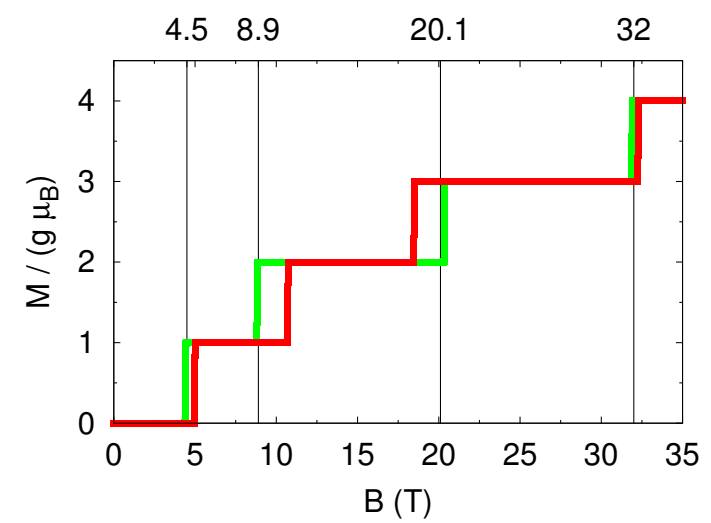

Fig. 11. Magnetization of the Hubbard model $\widehat{\mathcal{H}}_{H}$ as a function of the applied magnetic field $B$ at $T=0.01 \mathrm{~K}$ for the parameters $t=3.91 \mathrm{meV}, U=64.6 \mathrm{meV}$, and $g=2.25$ (red/dark). The steps occur at magnetic fields of 5.0, 10.7, 18.5, and 32.3 T. For comparison, also the fit result of Ref. 45] is plotted (green/light), which was obtained with the Hamiltonian $\widehat{\mathcal{H}}_{e x}+\widehat{\mathcal{H}}_{2}+\widehat{\mathcal{H}}_{3}+\widehat{\mathcal{H}}_{B}$ (see section 4.3). The positions of the experimentally determined crossing fields are indicated by vertical lines.

the parameter space of the Hubbard model $\widehat{\mathcal{H}}_{H}$ is only two-dimensional, we have to conclude that $\widehat{\mathcal{H}}_{H}$ cannot explain the low-temperature magnetization in $\mathrm{Ni}_{4} \mathrm{Mo}_{12}$. Accordingly, it does not represent an adequate model for $\mathrm{Ni}_{4} \mathrm{Mo}_{12}$, and thus does not capture the physical origin of the non-Heisenberg interactions in the molecule. This clearly contradicts one of the main findings of Ref. 45. In the following we will hence substantiate our conclusion and clarify the situation. As a byproduct, we will identify an error in the analytical derivation of the spin model $\widehat{\mathcal{H}}_{e x}+\widehat{\mathcal{H}}_{2}+\widehat{\mathcal{H}}_{3}+\widehat{\mathcal{H}}_{B}$ in Ref. [45].

Before doing so, we should first comment on the proposed Hubbard model $\widehat{\mathcal{H}}_{H}$ and point out some of its shortcomings and possible modifications. As far as we understand it, the intra-ion hopping terms were neglected in Ref. [45] on the grounds that the Pauli principle prohibits a hopping between lattice sites which are occupied by electrons with equal spin projection. However, this argument is valid only in a classical picture of a spin-1 state. Quantum mechanically there is no a priori reason for neglecting the intra-ion hopping, because a level with $s=1$ can also have a magnetic quantum number of $m=0$. Neglecting the intra-ion hopping is hence an inadequate method to incorporate the known ground-state spin configuration of the Ni ions into the Hubbard model (see below). We have checked for a number of parameter sets that a non-zero value of $t_{a}$ can influence the low-temperature magnetization curve by shifting the position of the ground state energies in subspaces with fixed magnetic quantum number. In our fits to the magnetization data we therefore also added $t_{a}$ as a third independent fit parameter. However, this did not lead to any improvement. Furthermore, according to the motivation for using a Hubbard model as presented before, one should actually use a two-band Hubbard model because every $\mathrm{Ni}$ ion provides two magnetic orbitals. In the usual derivation of the Hubbard model (see e.g. Ref. 67]) this leads to a variety of additional interaction terms (intra-ion Coulomb repulsion, intra-ion exchange interaction, pair jumps, and correlated hopping). Of these we considered only the terms which are usually studied in the literature, i.e., longer-range Coulomb repulsion and Heisenberg exchange (extended Hubbard model). The Heisenberg exchange term provides a proper means to handle the spin state of the Ni ions, through the use of a ferromagnetic intra-ion coupling (Hund's rule coupling). Perhaps not surprisingly, the inclusion of further interaction terms allowed for a better fit of the magnetization data and improved the $\chi^{2}$ value by about one order of magnitude. However, this is still one order of magnitude worse than the fit result obtained in Ref. [45]. We thus do not believe that such generalizations of the Hubbard model $\widehat{\mathcal{H}}_{H}$ lead in the right direction.

In order to resolve the apparent contradiction between our conclusion and that in Ref. [45], we reanalyzed the strong-coupling limit $U \gg|t|$ of the Hamiltonian $\widehat{\mathcal{H}}_{H}$. In this limit the hopping term can be treated as a perturbation, which at half-filling leads to an effective spin model $\widehat{\mathcal{H}}_{s}$ whose energy eigenvalues are supposed to approximate the low-energy spectrum of the full Hubbard model [67. It should be noted that the effective Hamiltonian $\widehat{\mathcal{H}}_{s}$ is a spin-1/2 model, consisting of 8 spins in the case of $\mathrm{Ni}_{4} \mathrm{Mo}_{12}$. We calculated $\widehat{\mathcal{H}}_{s}$ analytically up to order $\mathcal{O}\left(t^{4} U^{-3}\right)$ using two approaches. Starting from the results of the so called Canonical Transformation [55, 68, Hubbard-X operators [69] were employed to rewrite the effective Hamiltonian in the form of a pure spin model, as in Ref. 70. Alternatively, one can use the general results of Takahashi [71] and MacDonald et al. [72] to directly find the effective spin Hamiltonian, which considerably speeds up the whole calculation. Both approaches lead to equivalent results. Denoting the spin Hamiltonian up to order $\mathcal{O}\left(t^{2} U^{-1}\right)$ as $\widehat{\mathcal{H}}_{s}^{(2)}$, and the one up to order $\mathcal{O}\left(t^{4} U^{-3}\right)$ as $\widehat{\mathcal{H}}_{s}^{(4)}$, we obtained the following expressions:

$$
\begin{aligned}
\widehat{\mathcal{H}}_{s}^{(2)}= & -j^{(2)} \sum_{\alpha<\beta}^{8} \hat{\boldsymbol{s}}_{\alpha} \cdot \hat{\boldsymbol{s}}_{\beta}-\frac{24 t^{2}}{U}+g \mu_{B} \hat{\boldsymbol{S}} \cdot \boldsymbol{B} \\
\widehat{\mathcal{H}}_{s}^{(4)}= & -j^{(4)} \sum_{\alpha<\beta}^{8} \hat{\boldsymbol{s}}_{\alpha} \cdot \hat{\boldsymbol{s}}_{\beta}-j_{i o n} \sum_{i=1}^{4} \hat{\boldsymbol{s}}_{\alpha_{i}} \cdot \hat{\boldsymbol{s}}_{\beta_{i}} \\
& -j_{4} \sum_{(\alpha \beta \gamma \delta)}\left[\left(\hat{\boldsymbol{s}}_{\alpha} \cdot \hat{\boldsymbol{s}}_{\beta}\right)\left(\hat{\boldsymbol{s}}_{\gamma} \cdot \hat{\boldsymbol{s}}_{\delta}\right)\right. \\
& \left.\quad+\left(\hat{\boldsymbol{s}}_{\alpha} \cdot \hat{\boldsymbol{s}}_{\delta}\right)\left(\hat{\boldsymbol{s}}_{\beta} \cdot \hat{\boldsymbol{s}}_{\gamma}\right)-\left(\hat{\boldsymbol{s}}_{\alpha} \cdot \hat{\boldsymbol{s}}_{\gamma}\right)\left(\hat{\boldsymbol{s}}_{\beta} \cdot \hat{\boldsymbol{s}}_{\delta}\right)\right] \\
& -\left(\frac{24 t^{2}}{U}-\frac{78 t^{4}}{U^{3}}\right)+g \mu_{B} \hat{\boldsymbol{S}} \cdot \boldsymbol{B} .
\end{aligned}
$$

The terms $-j^{(2)} \sum_{\alpha<\beta}^{8} \hat{\boldsymbol{s}}_{\alpha} \cdot \hat{\boldsymbol{s}}_{\beta}$ and $-j^{(4)} \sum_{\alpha<\beta}^{8} \hat{\boldsymbol{s}}_{\alpha} \cdot \hat{\boldsymbol{s}}_{\beta}$ describe the inter-ion interactions, where the sites $\alpha$ and $\beta$ belong to different ions; the term $-j_{i o n} \sum_{i=1}^{4} \hat{\boldsymbol{s}}_{\alpha_{i}} \cdot \hat{\boldsymbol{s}}_{\beta_{i}}$ describes the intra-ion interactions, where the sites $\alpha_{i}$ and $\beta_{i}$ belong to the same $\mathrm{Ni}$ ion $i\left[\left(\alpha_{i}, \beta_{i}\right)=(1,2),(3,4)\right.$, 
$(5,6),(7,8)$ for $i=1,2,3,4] .(\alpha \beta \gamma \delta)$ denotes the tetragon with vertices $\alpha, \beta, \gamma$, and $\delta$, which have to be connected by hopping paths such that $\alpha$ is connected to $\beta, \beta$ to $\gamma$, $\gamma$ to $\delta$, and $\delta$ to $\alpha$ (see also Fig. 10). The Zeeman term $g \mu_{B} \hat{\boldsymbol{S}} \cdot \boldsymbol{B}$ is unaffected in the perturbation theory as the total spin $\hat{\boldsymbol{S}}$ commutes with the hopping term. The coupling parameters were obtained as

$$
\begin{aligned}
& j^{(2)}=-\frac{4 t^{2}}{U}, \\
& j^{(4)}=-\frac{4 t^{2}}{U}+\frac{92 t^{4}}{U^{3}}, j_{4}=-\frac{80 t^{4}}{U^{3}}, \quad j_{i o n}=\frac{36 t^{4}}{U^{3}} .
\end{aligned}
$$

Corrections to the spin Hamiltonian $\widehat{\mathcal{H}}_{s}^{(4)}$ and the given parameters are of order $\mathcal{O}\left(t^{6} U^{-5}\right)$ 71. Even though intraion hoppings are not included in the model, a non-zero and ferromagnetic coupling between the spins belonging to the same $\mathrm{Ni}$ ion is obtained, $j_{i o n}>0$. However, it is of order $\mathcal{O}\left(t^{4} U^{-3}\right)$ and thus usually weak. We mention that the energy of the ferromagnetic ground state with $|M|=\left|M_{\text {max }}\right|$ is zero for both $\widehat{\mathcal{H}}_{H}$ and its effective spin Hamiltonians [the corresponding constants were explicitly retained in Eqs. (4)].

In order to evaluate the accuracy of our analytical results for $\widehat{\mathcal{H}}_{s}^{(2)}$ and $\widehat{\mathcal{H}}_{s}^{(4)}$, we calculated the energy eigenvalues of the different models for a number of parameter sets $t$ and $U$ via numerical exact diagonalization and compared the obtained energy spectra. For the ratio $U / t=20$ the energy spectra are presented in Fig. $12(t=1)$. Apparently, the spectrum of $\widehat{\mathcal{H}}_{s}^{(2)}$ clearly deviates from the exact spectrum, in particular at low energies, whereas the fourth-order Hamiltonian $\widehat{\mathcal{H}}_{s}^{(4)}$ gives a much better approximation. We found that for $U / t=30$ the low-energy spectrum of the Hubbard model $\widehat{\mathcal{H}}_{H}$ is already very well approximated by the eigenvalues of $\widehat{\mathcal{H}}_{s}^{(4)}$. However, we also observed that for a ratio $U / t=10$, which was inferred and identified with the strong-coupling limit $U \gg|t|$ in Ref. 45, the exact and fourth-order spectrum have little in common. A link between exact and approximative levels may be established starting with $U / t \approx 15$, but at this ratio clear discrepancies are still present, especially with respect to the ground state energy. In the following we will only consider $\widehat{\mathcal{H}}_{s}^{(4)}$.

Having established an effective spin Hamiltonian for the electron spins $\hat{\boldsymbol{s}}_{\alpha}$, we now discuss how to derive an effective spin model in the space of the Ni spins $\hat{\boldsymbol{S}}_{i}$. The total spin of the Ni ion $i$ is $\hat{\boldsymbol{S}}_{i}=\hat{\boldsymbol{s}}_{\alpha_{i}}+\hat{\boldsymbol{s}}_{\beta_{i}}$. Since the operators $\hat{\boldsymbol{S}}_{i}$ commute with $\widehat{\mathcal{H}}_{s}^{(4)}$, the quantum numbers $S_{i}$ are good quantum numbers. The part of the energy spectrum with $S_{i}=1$ for all $i$ can thus be extracted by adding a ferromagnetic coupling between the electron spins $\hat{\boldsymbol{s}}_{\alpha_{i}}$ and $\hat{\boldsymbol{s}}_{\beta_{i}}$ residing on the same Ni ion to $\widehat{\mathcal{H}}_{s}^{(4)}$ (Hund's rule coupling). Technically, this can be done by setting $j_{\text {ion }} \rightarrow \infty$ (energy levels with $S_{i}=0$ for at least one $i$ are lifted to arbitrarily high energies). However, it is important to note that this approach is only possible within the effective spin model. In the case of the Hubbard model $\widehat{\mathcal{H}}_{H}$

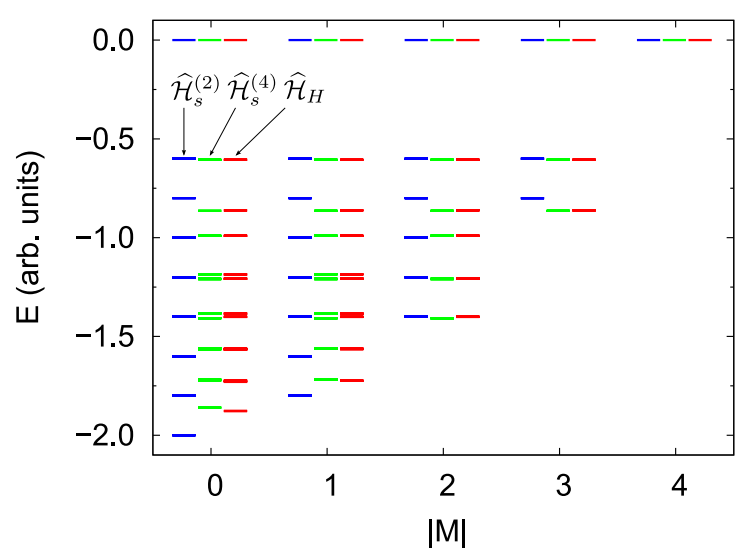

Fig. 12. Numerical comparison of the low-energy spectrum of the Hubbard model $\widehat{\mathcal{H}}_{H}$ (red/dark, right) and the complete spectrum of the spin model $\widehat{\mathcal{H}}_{s}^{(2)}$ (blue/dark, left) and $\widehat{\mathcal{H}}_{s}^{(4)}$ (green/light, middle) for the parameters $t=1$ and $U=20$. The energy eigenvalues are plotted vs. the absolute value of the total magnetic quantum number $|M|$. The product state with $|M|=4$ is an eigenstate of all three models with energy $E=0$.

the $S_{i}$ are not good quantum numbers since any operator which is specific to particular Hubbard lattice sites, like $\hat{\boldsymbol{S}}_{i}=\hat{\boldsymbol{s}}_{\alpha_{i}}+\hat{\boldsymbol{s}}_{\beta_{i}}$, cannot be a conserved quantity in an itinerant model. Adding a Hund's rule coupling directly to $\widehat{\mathcal{H}}_{H}$ would thus make the configurations with $S_{i}=1$ energetically favorable, but it would also change the structure of the whole energy spectrum. This means that it is incorrect to state that the spin model which is obtained by adding such a term to $\widehat{\mathcal{H}}_{s}^{(4)}$ follows from the original Hubbard model.

To carry out the limit $j_{\text {ion }} \rightarrow \infty$, the operators $\hat{\boldsymbol{S}}_{i}$ can be interpreted as pure spin-1 operators in a low-energy theory. The resulting spin model then becomes (using notation introduced in section 3 )

$$
\begin{aligned}
\widehat{\mathcal{H}}= & \widehat{\mathcal{H}}_{e x}+\widehat{\mathcal{H}}_{2}+\widehat{\mathcal{H}}_{3}+\widehat{\mathcal{H}}_{B} \\
& -J_{4} \sum_{(i j k l) \in \Gamma}\left(\hat{\boldsymbol{S}}_{i} \cdot \hat{\boldsymbol{S}}_{j}\right)\left(\hat{\boldsymbol{S}}_{k} \cdot \hat{\boldsymbol{S}}_{l}\right)-\left(\frac{24 t^{2}}{U}+\frac{288 t^{4}}{U^{3}}\right)
\end{aligned}
$$

with the coupling parameters

$$
J=-\frac{4 t^{2}}{U}+\frac{192 t^{4}}{U^{3}}, J_{2}=J_{3}=\frac{J_{4}}{2}=-\frac{40 t^{4}}{U^{3}},
$$

and $\Gamma=\{(1,2,3,4),(1,3,2,4),(1,4,2,3)\}$. We mention that introducing an intra-ion hopping of strength $t_{a}$ $=t\left(\right.$ instead of $\left.t_{a}=0\right)$ in $\widehat{\mathcal{H}}_{H}$ does not change the Hamiltonian Eq. (6) (but certainly changes $\widehat{\mathcal{H}}_{s}^{(2)}$ and $\widehat{\mathcal{H}}_{s}^{(4)}$ ), which is due to the high symmetry of the present case. These results finally clarify our different findings for the magnetization steps when calculated with the Hubbard model $\widehat{\mathcal{H}}_{H}$ and the spin model $\widehat{\mathcal{H}}_{e x}+\widehat{\mathcal{H}}_{2}+\widehat{\mathcal{H}}_{3}+\widehat{\mathcal{H}}_{B}$, and should be compared to those in Ref. 45. One notable difference is that the Hamiltonian Eq. (6) comprises 4-spin interactions. Starting from Eq. (4b), it can easily be verified 
that such terms have to appear in the projection of $\widehat{\mathcal{H}}_{s}^{(4)}$ onto the subspace with $S_{i}=1$ for all $i$. Furthermore, we find that the parameters $J_{2}$ and $J_{3}$ are of equal strength, whereas in Ref. 45 they differ by a factor of 2 . Because the analysis of the experimental magnetization data with the spin model $\widehat{\mathcal{H}}_{e x}+\widehat{\mathcal{H}}_{2}+\widehat{\mathcal{H}}_{3}+\widehat{\mathcal{H}}_{B}$ yielded such a factor of 2 and the inferred ratio $U / t=10$, it was concluded that the Hubbard model provides a valid description of the magnetism in $\mathrm{Ni}_{4} \mathrm{Mo}_{12}$ [73. However, our results demonstrate that such a conclusion is not supported.

\section{Conclusion}

In conclusion, we presented inelastic neutron scattering data for the $\mathrm{Ni}_{4} \mathrm{Mo}_{12}$ molecule. An extensive analysis of these data in terms of a phenomenological spin Hamiltonian did not lead to a satisfactory description of its magnetism. A similar observation was made in previous works, mainly based on magnetization data 44. Confirming this observation by complementary spectroscopic data, in our opinion, significantly furthers the idea that for $\mathrm{Ni}_{4} \mathrm{Mo}_{12}$ the spin Hamiltonian approach is indeed inadequate. As an alternative and more fundamental model the Hubbard model comes to mind, which we hence analyzed in great detail. We have shown that the first-guess Hubbard model $\widehat{\mathcal{H}}_{H}$ cannot explain the magnetization data of $\mathrm{Ni}_{4} \mathrm{Mo}_{12}$ as it predicts incorrect crossing fields. Obvious extensions of the model did not resolve the issue satisfactorily either. We then studied the strong-coupling limit of $\widehat{\mathcal{H}}_{H}$ in order to better understand these results. According to our calculations, the observation in Ref. 45 that the magnetization curve can be nicely fitted with the spin model $\widehat{\mathcal{H}}_{e x}+\widehat{\mathcal{H}}_{2}+\widehat{\mathcal{H}}_{3}+\widehat{\mathcal{H}}_{B}$ does not imply that the Hubbard model is a suitable microscopic model for $\mathrm{Ni}_{4} \mathrm{Mo}_{12}$. In this work we have in fact shown that the opposite is true.

\section{Acknowledgment}

We gratefully acknowledge financial support by the Deutsch Forschungsgemeinschaft and FOR 945.

\section{References}

1. D. Gatteschi, R. Sessoli, J. Villain, Molecular Nanomagnets (Oxford University Press, Oxford, 2006).

2. J. R. Friedman, M. P. Sarachik, J. Tejada, R. Ziolo, Phys. Rev. Lett. 76, 3830 (1996).

3. W. Wernsdorfer, R. Sessoli, Science 284, 133 (1999).

4. J. J. Borras-Almenar, J. M. Clemente-Juan, E. Coronado, B. S. Tsukerblat, Inorg. Chem. 38, 6081 (1999).

5. K. L. Taft, C. D. Delfs, G. C. Papaefthymiou, S. Foner, D. Gatteschi, S. J. Lippard, J. Am. Chem. Soc. 116, 823 (1994).

6. D. Gatteschi, A. Caneschi, L. Pardi, R. Sessoli, Science 265, 1054 (1994).

7. B. Pilawa, R. Desquiotz, M.T. Kelemen, M. Weickenmeier, A. Geisselman, J. Magn. Magn. Mater. 177, 748 (1997).
8. O. Waldmann, Coord. Chem. Rev. 249, 2550 (2005).

9. D. M. Low, G. Rajaraman, M. Helliwell, G. Timco, J. van Slageren, R. Sessoli, S. T. Ochsenbein, R. Bircher, C. Dobe, O. Waldmann, H. U. Güdel, M. A. Adams, E. Ruiz, S. Alvarez, E. J. L. McInnes, Chem. Eur. J. 12, 1385 (2006).

10. R. E. P. Winpenny, Adv. Inorg. Chem. 52, 1 (2003).

11. A. L. Dearden, S. Parsons, R. E. P. Winpenny, Angew. Chem. Int. Ed. 40, 151 (2001).

12. A. Cornia, M. Affronte, A. G. M. Jansen, G. L. Abbati, D. Gatteschi, Angew. Chem. Int. Ed. 38, 2264 (1999).

13. O. Waldmann, J. Schülein, R. Koch, P. Müller, I. Bernt, R. W. Saalfrank, H. P. Andres, H. U. Güdel, P. Allenspach, Inorg. Chem. 38, 5879 (1999).

14. R. W. Saalfrank, I. Bernt, E. Uller, F. Hampel, Angew. Chem. Int. Ed. Engl. 36, 2482 (1997).

15. J. van Slageren, R. Sessoli, D. Gatteschi, A. A. Smith, M. Helliwell, R. E. P. Winpenny, A. Cornia, A.-L. Barra, A. G. M. Jansen, E. Rentschler, G. A. Timco, Chem. Eur. J. 8, 277 (2002).

16. H. C. Yao, J. J. Wang, Y. S. Ma, O. Waldmann, W. X. Du, Y. Song, Y. Z. Li, L. M. Zheng, S. Decurtins, X. Q. Xin, Chem. Commun. 16, 1745-1747 (2006).

17. P. King, T. C. Stamatatos, K. A. Abboud, G. Christou, Angew. Chem. Int. Ed. Engl. 45, 7379 (2006).

18. K. Bärwinkel, P. Hage, H.-J. Schmidt, J. Schnack, Phys. Rev. B 68, 054422 (2003).

19. O. Waldmann, T. Guidi, S. Carretta, C. Mondelli, A. L. Dearden, Phys. Rev. Lett. 91, 237202 (2003).

20. P. W. Anderson, Basic Notions of Condensed Matter Physics (Benjamin/Cummings Publishing Co., Menlo Park, 1984).

21. B. Bernu, C. Lhuillier, and L. Pierre, Phys. Rev. Lett. 69, 2590 (1992).

22. O. Waldmann, Phys. Rev. B 65, 024424 (2001).

23. E. M. Chudnovsky, J. Tejada, Macroscopic Quantum Tunneling of the Magnetic Moment (Cambridge University Press, Cambridge, 1998).

24. B. Barbara, E. Chudnovsky, Phys. Lett. A 145, 205 (1990). 25. A. Chiolero, D. Loss, Phys. Rev. Lett. 80, 169 (1998).

26. O. Waldmann, T. C. Stamatatos, G. Christou, H. U. Güdel, I. Sheikin, H. Mutka, Phys. Rev. Lett. 102, 157202 (2009).

27. D. Gatteschi, L. Pardi, Gazz. Chim. Ital. 123, 231 (1993).

28. O. Waldmann, Phys. Rev. B 61, 6138 (2000).

29. A. Müller, S. Sarkar, S. Q. N. Shah, H. Bögge, M. Schmidtmann, P. Kögerler, B. Hauptfleisch, A. Trautwein, V. Schünemann, Angew. Chem. Int. Ed. 38, 3238 (1999).

30. U. Kortz, A. Müller, J. van Slageren, J. Schnack, N. S. Dalal, M. Dressel, Coord. Chem. Rev. 253, 2315 (2009).

31. C. Schröder, H. Nojiri, J. Schnack, P. Hage, M. Luban, P. Kögerler, Phys. Rev. Lett. 94, 017205 (2005).

32. I. Rousochatzakis, A. M. Läuchli, F. Mila, Phys. Rev. B 77, 094420 (2008)

33. R. Schmidt, J. Schnack, J. Richter, J. Magn. Magn. Mater. 295, 164 (2005).

34. J. Schnack, M. Luban, R. Modler, Europhys. Lett. 56, 863 (2001).

35. M. Exler, J. Schnack, Phys. Rev. B 67, 094440 (2003).

36. R. Schenker, H. Weihe, H. U. Güdel, Inorg. Chem. 40, 4319 (2001).

37. R. Schenker, M. N. Leuenberger, G. Chaboussant, H. U. Güdel, and D. Loss, Chem. Phys. Lett. 358, 413 (2002). 
38. R. Schenker, M. N. Leuenberger, G. Chaboussant, D. Loss, H. U. Güdel, Phys. Rev. B 72, 184403 (2005).

39. O. Zaharko, J. Mesot, L. A. Salguero, R. Valentí, M. Zbiri, M. Johnson, Y. Filinchuk, B. Klemke, K. Kiefer, M. Mys'kiv, Th. Strässle, H. Mutka, Phys. Rev. B 77, 224408 (2008).

40. A. Sieber, C. Boskovic, R. Bircher, O. Waldmann, S. T. Ochsenbein, G. Chaboussant, H. U. Güdel, N. Kirchner, J. van Slageren, W. Wernsdorfer, A. Neels, H. Stoeckli-Evans, S. Janssen, F. Juranyi, H. Mutka, Inorg. Chem. 44, 43154325 (2005).

41. E. C. Yang, W. Wernsdorfer, L. N. Zakharov, Y. Karaki, A. Yamaguchi, R. M. Isidro, G. D. Lu, S. A. Wilson, A. L. Rheingold, H. Ishimoto, D. N. Hendrickson, Inorg. Chem. 45, 529-546 (2006).

42. E. del Barco, A. D. Kent, E. C. Yang, D. N. Hendrickson, Phys. Rev. Lett. 93, 157202 (1997).

43. A. Müller, C. Beugholt, P. Kögerler, H. Bögge, S. Budko, M. Luban, Inorg. Chem. 39, 5176 (2000).

44. J. Schnack, M. Brüger, M. Luban, P. Kögerler, E. Morosan, R. Fuchs, R. Modler, H. Nojiri, R. C. Rai, J. Cao, J. L. Musfeldt, X. Wei, Phys. Rev. B 73, 094401 (2006).

45. V. Kostyuchenko, Phys. Rev. B 76, 212404 (2007).

46. R. Klemm, D. Efremov, Phys. Rev. B 77, 184410 (2008).

47. G. Chaboussant, A. Sieber, S. Ochsenbein, H. U. Güdel, M. Murrie, A. Honecker, N. Fukushima, B. Normand, Phys. Rev. B 70, 104422 (2004).

48. R. Bircher, G. Chaboussant, C. Dobe, H. U. Güdel, S. T. Ochsenbein, A. Sieber, O. Waldmann, Adv. Funct. Mater. 16, 209 (2006).

49. S. Carretta, P. Santini, G. Amoretti, T. Guidi, J. R. D. Copley, Y. Qiu, R. Caciuffo, G. Timco, R. E. P. Winpenny, Phys. Rev. Lett. 98, 167401 (2007).

50. M. B. Stone, F. Fernandez-Alonso, D. T. Adroja, N. S. Dalal, D. Villagrán, F. A. Cotton, S. E. Nagler, Phys. Rev. B 75, 214427 (2007).

51. M. H. Whangbo, H. J. Koo, D. Dai, J. Solid State Chem. 176, 417 (2003).

52. P. Mialane, C. Duboc, J. Marrot, E. Riviere, A. Dolbecq, F. Secheresse, Chem.-Eur. J. 12, 1950 (2006).

53. M. Hagiwara, L. P. Regnault, A. Zheludev, A. Stunault, N. Metoki, T. Suzuki, S. Suga, K. Kakurai, Y. Koike, P. Vorderwisch, J. H. Chung, Phys. Rev. Lett. 94, 177202 (2005).

54. J. Hubbard, Proc. R. Soc. Ser. A 276, 238 (1963).

55. A. H. MacDonald, S. M. Girvin, D. Yoshioka, Phys. Rev. B 37, 9753 (1988).

56. J. A. Farrar, F. Neese, P. Lappalainen, P. M. H. Kroneck, M. Saraste, W. G. Zumft, A. J. Thomson, J. Am. Chem. Soc. 118, 11501 (1996).

57. M. Brüger, Ph.D. thesis, Osnabrück University (2008).

58. I. Dzyaloshinsky, J. Phys. Chem. Solids 4, 241 (1958).

59. T. Moriya, Phys. Rev. 120, 91 (1960).

60. N. Kirchner, J. van Slageren, B. Tsukerblat, O. Waldmann, M. Dressel, Phys. Rev. B 78, 094426 (2008).

61. A. Messiah, Quantenmechanik (Walter de Gruyter, Berlin, New York, 1985)

62. O. Waldmann, Phys. Rev. B 68, 174406 (2003).

63. O. Waldmann, Phys. Rev. B 72, 094422 (2005).

64. R. Boca, Coord. Chem. Rev. 248, 757 (2004).

65. E. Liviotti, S. Carretta, G. Amoretti, J. Chem. Phys. 117, 3361 (2002).

66. S. Datta, O. Waldmann, A. D. Kent, V. A. Milway, L. K. Thompson, S. Hill, Phys. Rev. B 76, 052407 (2007).
67. F. H. Essler, H. Frahm, F. Göhmann, A. Klümper, V. E. Korepin, The One-Dimensional Hubbard Model (Cambridge University Press, 2005).

68. A. L. Chernyshev, D. Galanakis, P. Phillips, A. V. Rozhkov, A.-M. S. Tremblay, Phys. Rev. B 70, 235111 (2004).

69. J. Hubbard, Proc. Roy. Soc. London 285, 542 (1965).

70. P. Kakashvili, G. I. Japaridze, J. Phys.: Cond. Mat. 16, 5815 (2004).

71. M. Takahashi, J. Phys. C 10, 1289 (1977).

72. A. H. MacDonald, S. M. Girvin, D. Yoshioka, Phys. Rev. B 43, 6209 (1991).

73. At this point it is important to note that the Hamiltonian (6) and the Hubbard model $\widehat{\mathcal{H}}_{H}$ share the ground state energies in the subspaces with given $M$ in the interesting parameter regime, i.e., in the regime where $\widehat{\mathcal{H}}_{s}^{(4)}$ can be an approximation for $\widehat{\mathcal{H}}_{H}$. For this reason they should predict the same crossing fields at $T=0$ in this regime. So, while the projection onto the space with $S_{i}=1$ for all $i$ in general breaks the connection between the effective spin model and the full Hubbard model, it does actually not matter in case of the magnetization at $T=0$. 